Check for updates

Cite this: RSC Adv., 2017, 7, 55257

\title{
Facile synthesis of nitrogen-doped porous carbon for high-performance supercapacitors
}

\author{
Wang Yang, ${ }^{\text {ab }}$ Wu Yang, ${ }^{a}$ Lina Kong, ${ }^{a}$ Ailing Song ${ }^{a}$ and Xiujuan Qin*ab \\ Nitrogen-doped porous carbon (NCs) have been synthesized by direct pyrolysis of a mixture containing \\ melamine, iron nitrate and ethylene diamine tetraacetic acid (EDTA). Both the addition of melamine and \\ the carbonization temperature play critical roles in the formation of the unique structure. The results \\ demonstrate that NC-700 shows the best electrochemical performance compared to the other samples. \\ High specific capacitance (275 $\mathrm{F} \mathrm{g}^{-1}$ at $0.3 \mathrm{~A} \mathrm{~g}^{-1}$ ) is obtained in $6 \mathrm{M} \mathrm{KOH}$, and the capacitance still \\ maintains $202 \mathrm{~F} \mathrm{~g}^{-1}$ when tested at $10 \mathrm{~A} \mathrm{~g}^{-1}$ (ca. 73.5\% capacitance retention). Moreover, the NC-700 \\ also possesses good cycling stability with only a loss of $2.0 \%$ after 10000 cycles at $10 \mathrm{~A} \mathrm{~g}^{-1}$. The facile \\ preparation method and good electrochemical performance render NCs a promising candidate for \\ supercapacitor applications.
}

Received 21st October 2017

Accepted 29th November 2017

DOI: 10.1039/c7ra11646b

rsc.li/rsc-advances

carbon with ammonia, amines, or urea, or employing various nitrogen-containing synthetic polymers, biomass or biomass derivatives, and so on. ${ }^{21-24}$ However, the preparation of these existing nitrogen-doped carbon materials generally requires complex and time-consuming synthesis routes. Consequently, it is highly desirable to develop a facile strategy to effectively prepare heteroatom-doped carbon materials, which is crucial for widespread implementation.

Herein, we designed and synthesized nitrogen-doped porous carbon (NCs) through a simple method. Melamine was introducted into the chelate of EDTA and iron nitrate in the synthesis process, and the interaction between these three components at high temperature yielded the nitrogen species introduced into the carbon matrix. Quite intriguingly, the addition of melamine and the carbonization temperature can influence the formation of the unique structure. The prepared NC-700 present favorable pore structure, which is beneficial to rapid ion transport and effective ion adsorption. Furthermore, the effective heteroatom doping synergistically improve the physicochemical properties and the capacitive behavior. As a result, the NC-700 exhibits good electrochemical performance when employed as electrode material for supercapacitor.

\section{Experimental}

\subsection{Chemicals}

In this experiment, all chemicals were used as purchased without any further processing. Deionized water was employed throughout the experiment.

\subsection{Preparation of nitrogen-doped porous carbon}

The nitrogen-doped porous carbon (NCs) were prepared via a preliminary heating process followed by carbonization at high 
temperature. Typically, $1.86 \mathrm{~g}$ EDTA, $2.02 \mathrm{~g}$ iron nitrate and $0.63 \mathrm{~g}$ melamine were dispersed in $25 \mathrm{~mL}$ deionized water, respectively. Subsequently, the EDTA solution and melamine solution were added into the iron nitrate solution with vigorous stirring at $90{ }^{\circ} \mathrm{C}$ to form homogeneous gel. Then the gel was put into oven for 12 hours to obtain the precursor. Afterwards, the precursor was annealed in tube furnace under inert atmosphere at desired temperature $\left(700-900{ }^{\circ} \mathrm{C}\right)$ for $2 \mathrm{~h}$ with a heating rate of $5{ }^{\circ} \mathrm{C} \mathrm{min}^{-1}$. The obtained products were then washed with $15 \mathrm{wt} \% \mathrm{HCl}$ solution. Finally, the samples were filtered, washed with abundant distilled water, and dried at $80{ }^{\circ} \mathrm{C}$ overnight. The nitrogen-doped carbon samples were labeled as NC- $x$, where the $x$ refers to the carbonization temperature. Comparatively, the carbon sample without melamine was also prepared at $700{ }^{\circ} \mathrm{C}$ and was named as C-700.

\subsection{Material characterization}

The structure of as-prepared materials were measured by X-ray diffraction (XRD) on a Rigaku Smart Lab X-ray diffractometer operated at $40 \mathrm{kV}$ with $\mathrm{Cu} \mathrm{K} \alpha$ radiation at a scan rate of $5^{\circ} \mathrm{min}^{-1}$. The morphologies of all the samples were analyzed by high-resolution transmission electron microscopy (HRTEM, JEOL-2100F). Nitrogen adsorption and desorption isotherms were characterized by Micromeritics V-Sorb 2800P analyzer at 77 K. Pore size distribution was obtained by the Barrette-JoynerHalenda (BJH) method. Elemental analysis was analyzed by Xray photoelectron spectroscopy (XPS) with a Kratos XSAM-800 spectrometer with monochromatized $\mathrm{Al} \mathrm{K} \alpha$ radiation.

\subsection{Electrochemical measurement}

The electrochemical performance was tested in $6 \mathrm{M} \mathrm{KOH}$ electrolyte. $\mathrm{Hg} / \mathrm{HgO}$ electrode and active carbon electrode were used as the reference electrode and the counter electrode, respectively. To prepare the working electrode, NCs, acetylene black and PTFE were mixed at a weight ratio of $80: 15: 5$ to form the homogeneous slurry. Then, the slurry was continuously spread on current collectors of $1 \mathrm{~cm}^{2}$ nickel foam, and dried at $80^{\circ} \mathrm{C}$ for $12 \mathrm{~h}$. The total mass loading of each electrode was approximately $2 \mathrm{mg}$. The fabricated electrodes were pressed at $4 \mathrm{MPa}$ for $30 \mathrm{~s}$, and immersed in $6 \mathrm{M} \mathrm{KOH}$ electrolyte for $24 \mathrm{~h}$. The specific capacitance of the working electrodes in three-electrode system was obtained from the galvanostatic discharge process via $C=I \Delta t /(m \Delta V)$, where $C$ is the specific capacitance $\left(\mathrm{F} \mathrm{g}^{-1}\right), I$ (A) refers the discharge current, $\Delta t(\mathrm{~s})$ is the discharge time, $m$ $(\mathrm{g})$ is the mass of the active material for working electrode and $\Delta V(\mathrm{~V})$ is the potential change excluding the $I R$ drop during the discharge process.

The galvanostatic charge-discharge measurement was recorded on a NEWARE auto-cycler. The potential window was -1.0 to $0.2 \mathrm{~V}$ in $6 \mathrm{M} \mathrm{KOH}$ for the three-electrode system. Cyclic voltammetry (CV) and electrochemical impedance spectroscopy (EIS) measurements were conducted on a CHI660E electrochemical workstation (Chenhua, Shanghai, China). The frequency range for the EIS measurement was from $1 \mathrm{mHz}$ to 1 $\mathrm{MHz}$ with a perturbation amplitude of $5 \mathrm{mV}$.

\section{Results and discussion}

The general strategy for fabricating NC-700 is illustrated in Scheme 1. The nitrogen-doped porous carbon material was prepared by a novel method using nitrogen-containing carbon source. In a typical synthesis, the EDTA and iron ions were chelated under certain conditions, then the sol was formed after the evaporation of the solvent. In this process, the melamine was retained by the viscosity of the sol. After drying in oven, the precursor was carbonized at high temperature under inert atmosphere. Finally, the nitrogen porous carbon material was successfully prepared. In this strategy, iron can not only affect pore structure, but also can accelerate the catalytic graphitization of carbon materials. Simultaneously, the nitrogen atoms in EDTA and melamine can introduced into carbon rings in the carbonization process. Not surprisingly, the interaction between EDTA, iron and melamine results in the formation of the unique morphology.

Fig. 1(a-d) depicts the TEM images of the samples. Fig. 1(a) shows the TEM image of C-700, and it can be seen that the sample shows a matrix-type porous mesh structure without the addition of melamine. Clearly, the morphology of the NC-700 changes significantly after adding melamine in the preparation process (Fig. 1(b)). This may be attributed that the addition of melamine can prevent the re-polymerization of oligomeric molecules and impede the formation of three-dimensional network structure. Furthermore, the morphology of the

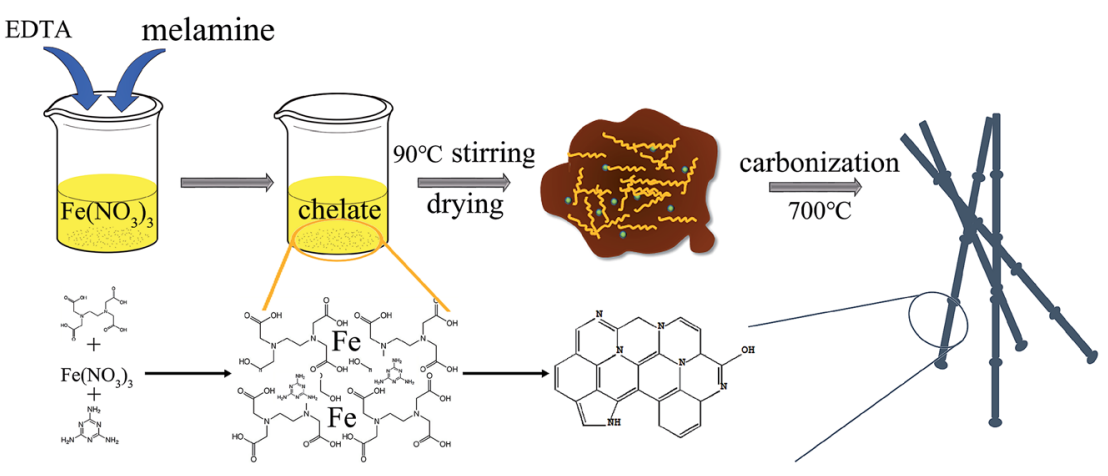

Scheme 1 Schematic representation of the fabrication process of NC-700. 

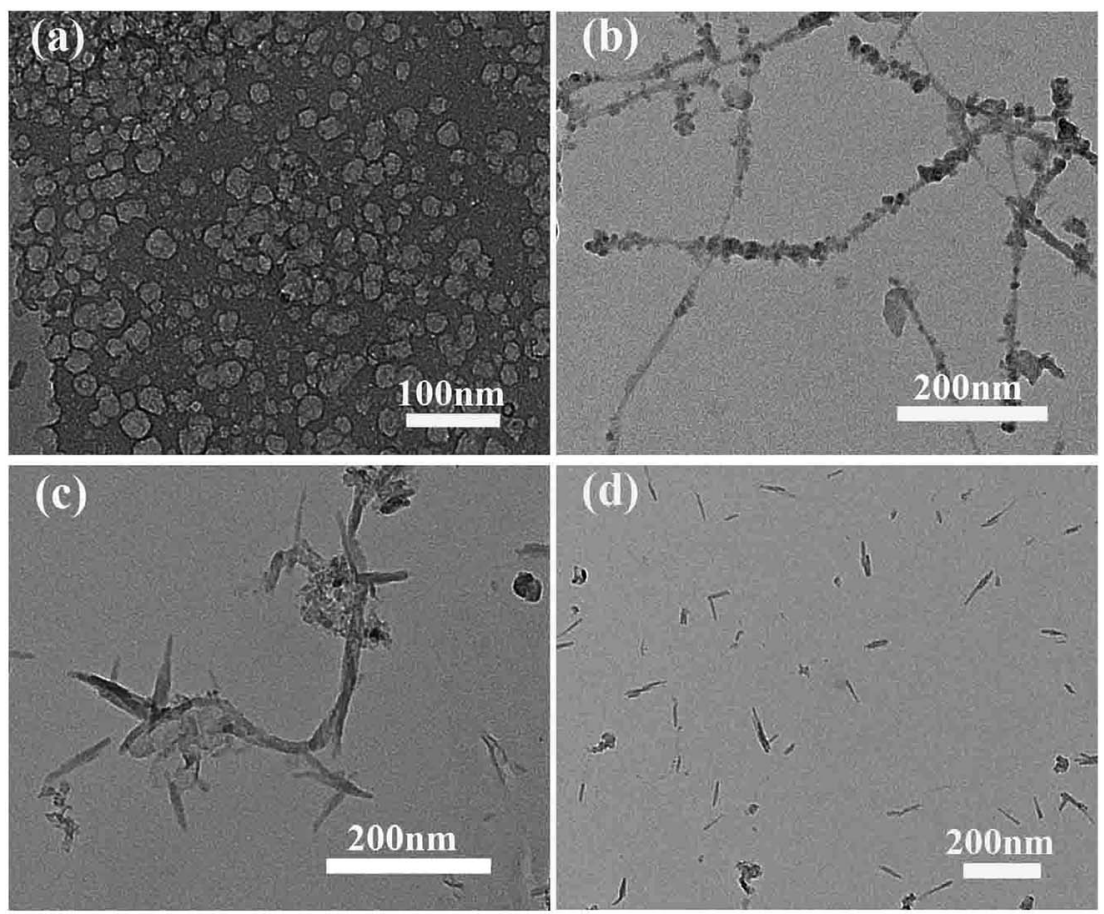

Fig. 1 (a-d) TEM image of the samples; (a) C-700; (b) NC-700; (c) NC-800; (d) NC-900.

material also changes along with the temperature varies from $700{ }^{\circ} \mathrm{C}$ to $900{ }^{\circ} \mathrm{C}$, as shown in Fig. 1(c and d). The above results exhibit that the unique structure becomes smoother and shorter as the carbonization temperature rises.

XRD patterns of the carbon samples are shown in Fig. 2(a). It can be seen that the sample without melamine (C-700) has a narrow peak near $26^{\circ}$ representing the (002) plane, ${ }^{25,26}$ which demonstrates a good graphitized structure. After adding melamine, the samples NC- $x$ have a large broad peak near $25^{\circ}$, indicating that melamine is not conducive to the graphitization of materials. The possible reason is ascribed that the chelating effect between EDTA and iron was reduced after adding of melamine, that is, smaller ratio of chelate containing iron was formed. Therefore, the catalytic graphitization in carbonation process is low. Moreover, it is obvious that the (002) peak becomes broader and weaker as the carbonization temperature increases, demonstrating that the graphitization degree of the material decreases. As a result, high temperature is also not beneficial for chelating effect between EDTA and iron.

Chemical composition of the carbon sample NC-700 was analysed in detail by X-ray photoelectron spectroscopy (XPS) in Fig. 2(b). The survey spectrum of the sample shows a predominant $\mathrm{C} 1 \mathrm{~s}$ peak at $284.5 \mathrm{eV}, \mathrm{O} 1 \mathrm{~s}$ peak at $532 \mathrm{eV}$ and $\mathrm{N} 1 \mathrm{~s}$ peak at $399 \mathrm{eV}^{29,30}$ The atomic percent of $\mathrm{C}, \mathrm{O}$ and $\mathrm{N}$ are $80 \%, 15.6 \%$ and $4.4 \%$, respectively. The possible advantages of the nitrogen doping are as following: (i) the $\mathrm{N}$ and $\mathrm{O}$ doping produced the heteroatom functional groups on the carbon surface, which is favorable for electrolyte soaking into the interior of material; (ii) some functional groups have a considerable effect on the electrochemical performance and can provide additional pseudocapacitance; (iii) the doping of nitrogen heteroatoms can improve the electrical conductivity of the material and reduce the internal impedance of the material. ${ }^{31,32}$ In short, the carbon material modified by nitrogen heteroatom can greatly improve the electrochemical properties of carbon electrode materials.

Fig. 2(c and d) presents $\mathrm{N}_{2}$ adsorption-desorption isotherm and the pore size distribution of the NC-700. According to IUPAC classification, ${ }^{27}$ the isotherm belongs to type IV corresponding to porous carbon with meso-pore size distribution. When the relative pressure is greater than 0.4 , there is a significant hysteresis loop, which indicates that the material has a mesoporous structure. When the relative pressure is greater than 0.9 , the curve shows a sudden increase trend, indicating that there is a gap between the particles of the material. The specific surface area of the material is $308.5 \mathrm{~m}^{2} \mathrm{~g}^{-1}$ and the pore volume is $0.59 \mathrm{~cm}^{3} \mathrm{~g}^{-1}$. Fig. 2(d) shows that the NC-700 has a hierarchical pore structure with micropores of less than $2 \mathrm{~nm}$ and mesopores with pore diameters of about $3.5 \mathrm{~nm}$. It is proved that micropores with less than $2 \mathrm{~nm}$ can provide a large area of adsorption sites for the accumulation of charge, which can facilitate the formation of electric double layer capacitance and improve the capacitance of electrode materials. The mesoporous about 3-4 $\mathrm{nm}$ provide an favorable path for the electrolyte ion diffusion, allowing the electrolyte ions to be transported quickly during the charge-discharge process at large current densities. ${ }^{28}$

To probe the electrochemical performance of the carbon samples, cyclic voltammetry (CV) and galvanostatic chargedischarge (GCD) were measured by three-electrode system in $6 \mathrm{M} \mathrm{KOH}$. Fig. 3(a) presents the CV curves of the samples at the scan rate of $20 \mathrm{mV} \mathrm{s}^{-1}$. Clearly, the NCs shows a slightly distorted rectangular $\mathrm{CV}$ curve with a wide reversible hump, 
(a)
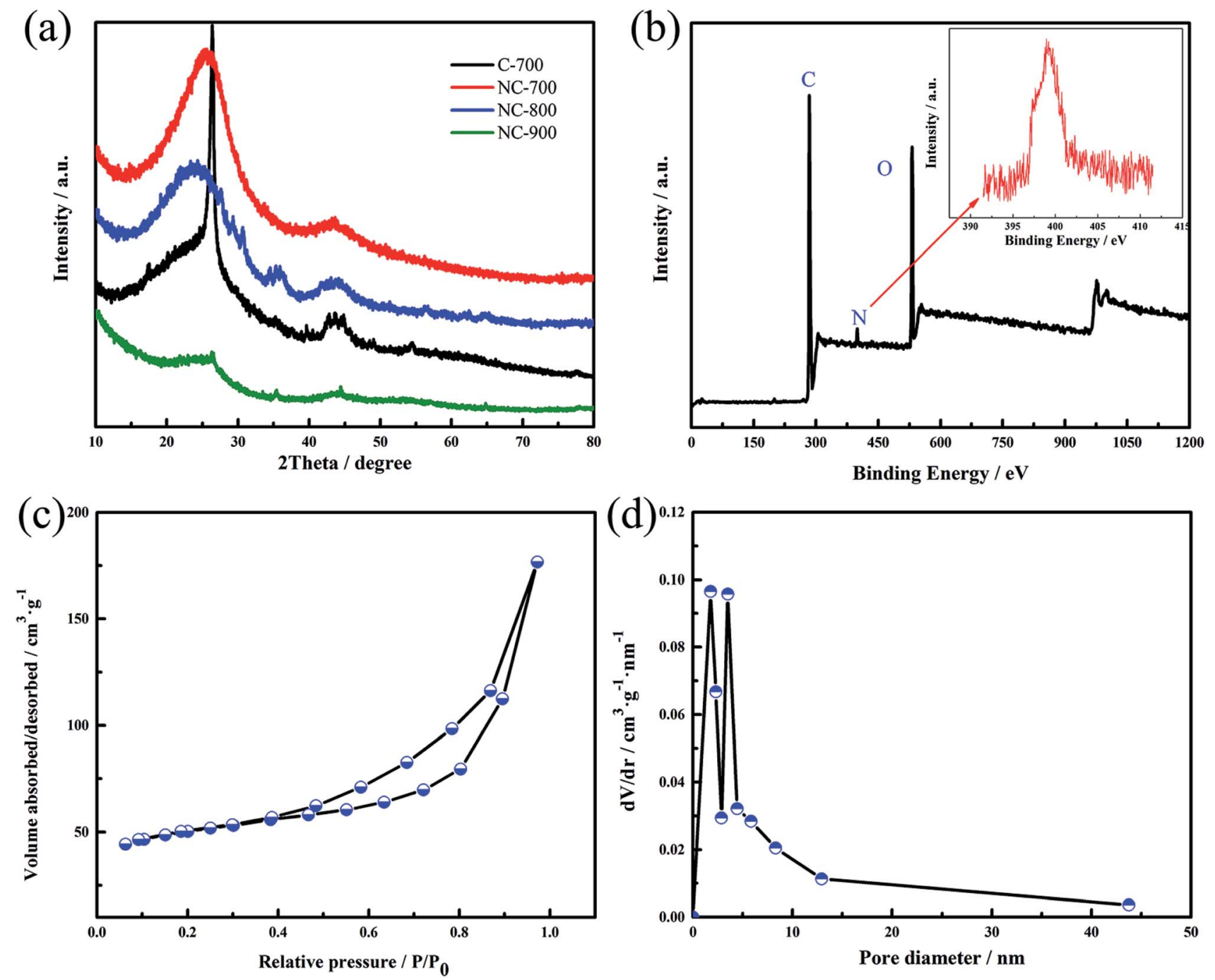

Fig. 2 (a) XRD patterns of the samples; (b) XPS survey spectrum of NC-700; (c) nitrogen adsorption-desorption isotherm of NC-700; (d) pore size distribution of the NC-700.

suggesting its electric double-layer capacitor (EDLC) performance along with the pseudocapacitance characteristics. ${ }^{33}$ The C-700 exhibits better-symmetric and rectangular CV curves, which can be ascribed to the absence of nitrogen doping. The GCD curves of the samples at $1.5 \mathrm{~A} \mathrm{~g}^{-1}$ (Fig. 3(b)) demonstrate that the NCs shows more non-linear characteristics than the C700 , further revealing the pseudo-capacitive feature generated from the doping elements, ${ }^{34}$ which is consistent with the $\mathrm{CV}$ result.

$\mathrm{CV}$ curves of NC-700 at different scan rates from $5 \mathrm{mV} \mathrm{s}^{-1}$ to $100 \mathrm{mV} \mathrm{s}^{-1}$ are presented in Fig. 4(a). We can see that the slightly distorted rectangular have no obvious change even at high scan rate, demonstrating its good rate performance. The same conclusion can be obtained from the GCD results of the NC-700 tested at different current densities from $0.3 \mathrm{~A} \mathrm{~g}^{-1}$ to $10 \mathrm{~A} \mathrm{~g}^{-1}$ (Fig. 4(b)). Fig. 4(c) exhibits the rate performance of the NC-700 with different current densities from $0.3 \mathrm{~A} \mathrm{~g}^{-1}$ to $10 \mathrm{~A} \mathrm{~g}^{-1}$. The specific capacitance calculated from the GCD curve is $275 \mathrm{~F} \mathrm{~g}^{-1}$ at $0.3 \mathrm{~A} \mathrm{~g}^{-1}$, and retains $202 \mathrm{~F} \mathrm{~g}^{-1}$ at $10 \mathrm{~A} \mathrm{~g}^{-1}$ (the retention is as high as $73.5 \%$ ). Such a good capacitance and rate performance is derived from the synergistic effect of porous structure and nitrogen doping: the mesopores facilitate electrolyte ion transport; the nitrogen doping provide pseudocapacitance and the functional groups can also improve the wettability of electrode material. ${ }^{35-37}$ The sample NC-700 also shows a good cycling performance at a current density of $10 \mathrm{~A} \mathrm{~g}^{-1}$ in Fig. 4(d). The specific capacitance retention after 10000 cycles is $98 \%$.

In order to further characterize the relationship between structure, chemical composition and electrochemical properties of the material, the electrochemical impedance spectroscopy (EIS) measurement was conducted shown in Fig. 5 . The Nyquist plots of C-700 show that there is no obvious semicircle in correlation with the charge-transfer resistance. ${ }^{38}$ The approximate vertical line at low frequency region further demonstrates the good capacitive characteristics. ${ }^{39}$ In addition, the inset shows that there is a significant semicircle in the high frequency region of $\mathrm{NC}-700$, and the semicircularity of the other 

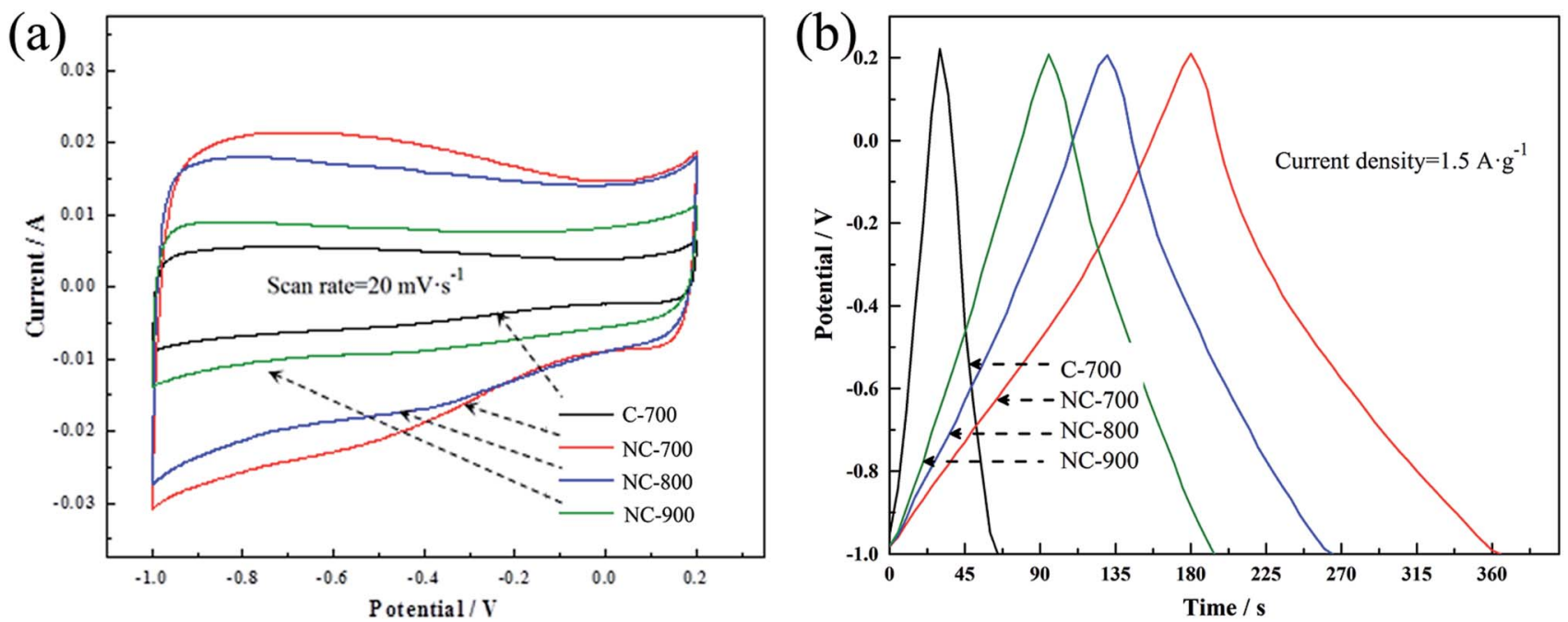

Fig. 3 (a) CV curves of the samples at the scan rate of $20 \mathrm{mV} \mathrm{s}^{-1}$; (b) galvanostatic charge-discharge curves of the samples measured at the current density of $1.5 \mathrm{~A} \mathrm{~g}^{-1}$.
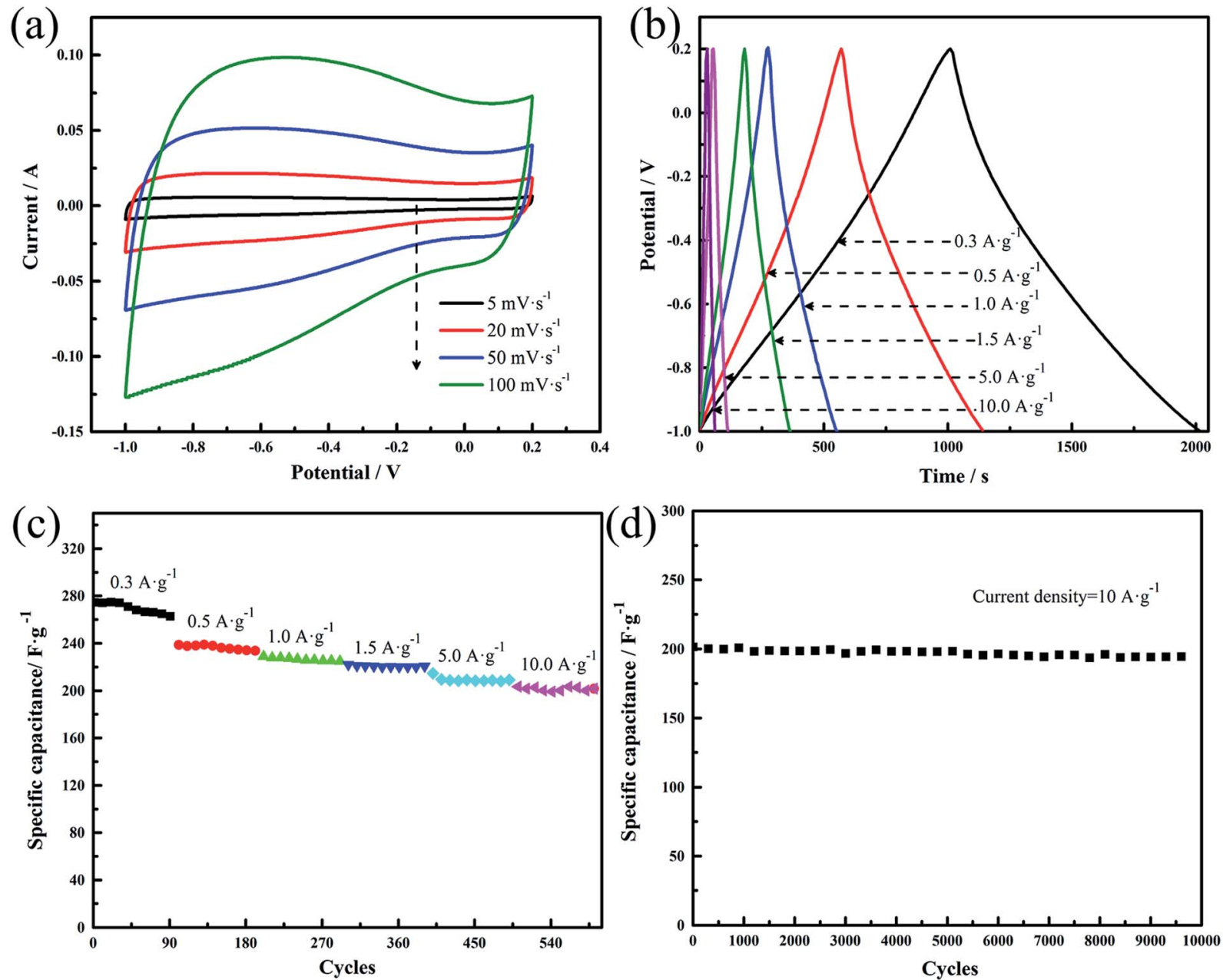

Fig. 4 (a) CV curves of NC-700 at different scan rates; (b) galvanostatic charge-discharge curves of NC-700 at different current densities; (c) specific capacitance calculated by discharge curves at various current densities; (d) cycling life of NC-700 tested at a current density of $10 \mathrm{Ag}^{-1}$ in a three-electrode system. 


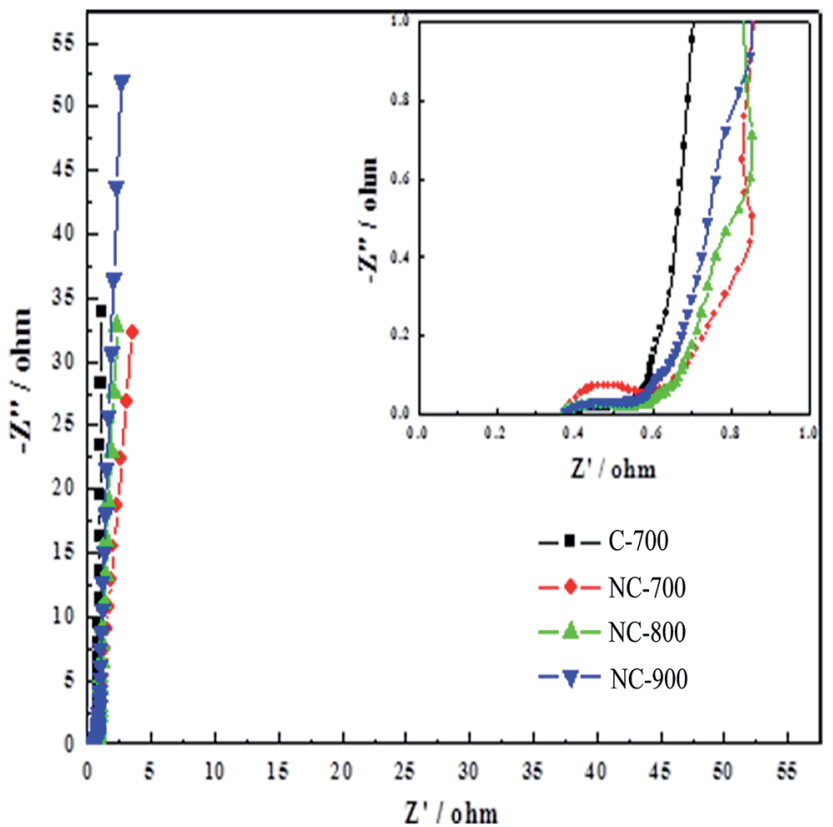

Fig. 5 Nyquist plots of the carbon samples (the inset shows the amplified part of the Nyquist plots).

samples is not significant, indicating that the NC-700 sample has a significant electrochemical reaction resistance and the most significant pseudo-capacitance behavior. As the carbonization temperature rises, NC-900 presents the most vertical line, that is, it delivers the best capacitive characteristics. Among all the samples, the C-700 shows the most vertical line, implying that the nitrogen doping is not advantageous to exhibit good capacitive characteristic due to the charge-transfer process.

\section{Conclusions}

In summary, nitrogen-doped porous carbon (NCs) were prepared by a facile method. Generally, EDTA and iron ion chelate can generate macromolecules. After adding melamine in the chelating process, the viscosity of macromolecules sol can retain melamine in the matrix during carbonation process, thus a nitrogen-doped porous carbon material was prepared. It was characterized by structural and electrochemical measurements, and the relationship between structure and electrochemical performance are discussed. The mesopores provide an favorable path for the electrolyte ion diffusion, allowing the electrolyte ions to be transported quickly during the chargedischarge process at large current densities. Moreover, the nitrogen doping has a considerable effect on the electrochemical performance and can provide additional pseudocapacitance. Consequently, the facile preparation method and synergistic effect between structure and nitrogen doping render NCs to be a promising candidate for supercapacitor application.

\section{Conflicts of interest}

There are no conflicts to declare.

\section{Acknowledgements}

We are grateful for the financial support from the National Natural Science Foundation of China (No. 51674221) and the Postgraduate Innovation Project of Hebei Province (No. CXZZBS2017058).

\section{References}

1 P. Simon, Y. Gogotsi and B. Dunn, Science, 2014, 343, 12101211.

2 Y. Wang, Y. Song and Y. Xia, Chem. Soc. Rev., 2016, 45, 59255950.

3 J. Yan, Q. Wang, T. Wei and Z. Fan, Adv. Energy Mater., 2014, 4, 1300816.

4 W. Yang, W. Yang, A. Song, L. Gao, G. Sun and G. Shao, J. Power Sources, 2017, 348, 175-182.

5 W. Yang, W. Yang, J. Feng, Z. Ma and G. Shao, Electrochim. Acta, 2016, 210, 71-78.

6 Y. Zhai, Y. Dou, D. Zhao, P. F. Fulvio, R. T. Mayes and S. Dai, Adv. Mater., 2011, 23, 4828-4850.

7 F. Béguin, V. Presser, A. Balducci and E. Frackowiak, $A d v$. Mater., 2014, 26, 2219-2251.

8 J. Wang, H. L. Xin and D. Wang, Part. Part. Syst. Charact., 2014, 31, 515-539.

9 C. Zhu, T. Liu, F. Qian, T. Y.-J. Han, E. B. Duoss, J. D. Kuntz, C. M. Spadaccini, M. A. Worsley and Y. Li, Nano Lett., 2016, 16, 3448-3456.

10 Z. Chen, Z. Ma, J. Song, L. Wang and G. Shao, J. Power Sources, 2016, 324, 86-96.

11 A. Song, W. Yang, W. Yang, G. Sun, X. Yin, L. Gao, Y. Wang, X. Qin and G. Shao, ACS Sustainable Chem. Eng., 2017, 5, 3973-3981.

12 M. Zhao, H. Dong, Z. Chen, Z. Ma, L. Wang, G. Wang, W. Yang and G. Shao, Int. J. Hydrogen Energy, 2016, 41, 20485-20493.

13 S. Huo, P. Duan, T. Jiao, Q. Peng and M. Liu, Angew. Chem., Int. Ed., 2017, 56, 12174-12178.

14 W. Yang, W. Yang, A. Song, L. Gao, L. Su and G. Shao, J. Power Sources, 2017, 359, 556-567.

15 W. Yang, W. Yang, L. Kong, A. Song, X. Qin and G. Shao, Carbon, 2018, 127, 557-567.

16 J. Pu, C. Li, L. Tang, T. Li, L. Ling, K. Zhang, Y. Xu, Q. Li and Y. Yao, Carbon, 2015, 94, 650-660.

17 Y. Li, G. Wang, T. Wei, Z. Fan and P. Yan, Nano Energy, 2016, 19, 165-175.

18 G. Wang, Y. Sun, D. Li, H.-W. Liang, R. Dong, X. Feng and K. Müllen, Angew. Chem., 2015, 127, 15406-15411.

19 H. Zhang, Y. Zhou, C. Li, S. Chen, L. Liu, S. Liu, H. Yao and H. Hou, Carbon, 2015, 95, 388-395.

20 K. Sun, E. Feng, H. Peng, G. Ma, Y. Wu, H. Wang and Z. Lei, Electrochim. Acta, 2015, 158, 361-367.

21 M. Zhou, F. Pu, Z. Wang and S. Guan, Carbon, 2014, 68, 185194.

22 L. Wan, E. Shamsaei, C. D. Easton, D. Yu, Y. Liang, X. Chen, Z. Abbasi, A. Akbari, X. Zhang and H. Wang, Carbon, 2017, 121, 330-336. 
23 J. Jiang, L. Bao, Y. Qiang, Y. Xiong, J. Chen, S. Guan and J. Chen, Electrochim. Acta, 2015, 158, 229-236.

24 W. Yang, Z. Du, Z. Ma, G. Wang, H. Bai and G. Shao, RSC Adv., 2016, 6, 3942-3950.

25 F. Su, C. K. Poh, J. S. Chen, G. Xu, D. Wang, Q. Li, J. Lin and X. W. Lou, Energy Environ. Sci., 2011, 4, 717-724.

26 W. Yang, W. Yang, F. Ding, L. Sang, Z. Ma and G. Shao, Carbon, 2017, 111, 419-427.

27 M. Thommes, K. Kaneko, A. V. Neimark, J. P. Olivier, F. Rodriguez-Reinoso, J. Rouquerol and K. S. Sing, Pure Appl. Chem., 2015, 87, 1051-1069.

28 Z. Li, Z. Xu, X. Tan, H. Wang, C. M. B. Holt, T. Stephenson, B. C. Olsen and D. Mitlin, Energy Environ. Sci., 2013, 6, 871878.

29 J. Wu, X. Zheng, C. Jin, J. Tian and R. Yang, Carbon, 2015, 92, 327-338.

30 Y. Mao, H. Duan, B. Xu, L. Zhang, Y. Hu, C. Zhao, Z. Wang, L. Chen and Y. Yang, Energy Environ. Sci., 2012, 5, 7950-7955.

31 Y. Li, C. Lu, S. Zhang, F.-Y. Su, W. Shen, P. Zhou and C. Ma, J. Mater. Chem. A, 2015, 3, 14817-14825.
32 T. Jawhari, A. Roid and J. Casado, Carbon, 1995, 33, 15611565.

33 L.-F. Chen, Z.-H. Huang, H.-W. Liang, H.-L. Gao and S.-H. Yu, Adv. Funct. Mater., 2014, 24, 5104-5111.

34 W. Yang, W. Yang, A. Song, L. Gao, L. Su and G. Shao, J. Power Sources, 2017, 359, 556-567.

35 H. Su, H. Zhang, F. Liu, F. Chun, B. Zhang, X. Chu, H. Huang, W. Deng, B. Gu, H. Zhang, X. Zheng, M. Zhu and W. Yang, Chem. Eng. J., 2017, 322, 73-81.

36 H. R. Byon, B. M. Gallant, S. W. Lee and Y. Shao-Horn, Adv. Funct. Mater., 2013, 23, 1037-1045.

37 Y. Zhang, T. Mori, J. Ye and M. Antonietti, J. Am. Chem. Soc., 2010, 132, 6294-6295.

38 P. Justin, S. K. Meher and G. R. Rao, J. Phys. Chem. C, 2010, 114, 5203-5210.

39 D. Saha, Y. Li, Z. Bi, J. Chen, J. K. Keum, D. K. Hensley, H. A. Grappe, H. M. Meyer, S. Dai, M. P. Paranthaman and A. K. Naskar, Langmuir, 2014, 30, 900-910. 